\title{
EDITORIAL \\ Direct versus indirect bypass for moyamoya disease: ongoing controversy
}

\author{
Mario K. Teo, MBChB, FRCS(SN), Venkatesh S. Madhugiri, MBBS, MCh, and \\ Gary K. Steinberg, MD, PhD
}

Department of Neurosurgery, Stanford University School of Medicine, Stanford, California

I $\mathrm{N}$ this issue of the Journal of Neurosurgery, Macyszyn and colleagues present a comparative analysis of direct, indirect, and combined revascularization techniques for moyamoya disease in adults and children based on data culled from the available English-language scientific literature. ${ }^{20}$ They conclude that outcomes after direct revascularization are significantly inferior compared with outcomes after the other two surgical approaches in both patient populations. The authors should be congratulated for attempting to address the controversy regarding the preferred revascularization procedure for moyamoya disease.

In our own experience, direct revascularization for moyamoya disease is a very well-established technique, and a number of authors have described excellent results in both adult and pediatric populations.,.$^{4,-9,13,14}$ The main advantages of direct anastomosis over indirect procedures are the ability to augment flow immediately after surgery, a more consistent and higher extent of angiographic collateralization, , 2,3,5,10,12 and superiority in restoring post-bypass cerebrovascular reserve capacity. ${ }^{5}$ Direct anastomoses are also associated with excellent clinical outcomes, including a higher rate of symptomatic improvement, lower risk of recurrent ischemia, and increased stroke-free survival compared with indirect bypass. ${ }^{1,11,12}$

In their comparative analysis of adult patients, the authors included 6 adult series (one of which was our Stanford series), in which 762 cases had direct bypasses and 1524 had indirect bypasses. The perioperative risks of death, ischemic stroke, and intracranial hemorrhage were very similar between the two groups. However, the long-term risk of ischemic stroke in the indirect bypass group (10.5\%) far exceeded that in the direct bypass group $(1.4 \%)$. Interestingly, the authors stated that meta-regression analysis showed no significant increased rate of stroke or hemorrhage with length of follow-up but did not quan- tify further with regards to which intervention group they were addressing. Additionally, they stated that "indirect revascularization results in over one-half a QALY more than the direct option during the 4-year follow-up. This difference is highly statistically significant $(\mathrm{p}<0.001)$." However, in analyzing the authors' Table 4, the expected QALYs in adults at the 4-year follow-up were 3.502 for the direct bypass group and 3.553 for the indirect bypass group; thus, a difference of a 0.05 QALY was obtained. Although this difference might be statistically significant, whether this translates into clinical significance is doubtful. Therefore, based on the data presented by the authors, we believe that direct bypass is still a better revascularization procedure in adults, especially in view of the lower long-term stroke risk.

With regard to the comparative analysis in the pediatric cohort, 34 series, including 1900 cases, were included (1526 indirect bypasses, 258 combined procedures, and 116 direct bypasses). The authors then concluded that combined and indirect approaches are both superior to direct revascularization based on the analysis performed in the pediatric population. However, we would like to reiterate that in combined procedures, patients undergo a direct and an indirect component of the revascularization in the same setting. Therefore, the direct component would provide an immediate increase in cerebral perfusion, while the indirect collateralization would take months to form. This is also the case in most direct procedures, wherein after direct superficial temporal artery to middle cerebral artery bypass, the perivascular cuff forms indirect collateralization as seen on follow-up angiograms. Therefore, in our opinion, a more suitable analysis would have been to include the direct and combined groups as a single group to compare with the indirect group, as reported by other authors. ${ }^{12}$

While going through the case series selected to perform 
this comparative analysis, we also would like to highlight inconsistencies. Several publications by Kim et al. were included ${ }^{15-19}$ and while some of the series were from different time intervals, there were patient cohorts that were counted multiple times, which were assumed to be different in view of the different publications. We also note that in some of the larger series, for example by Duan et al., ${ }^{6}$ in which the majority of the patients underwent indirect revascularization, no distinction in outcome was made based on the type of bypass. It is therefore unclear how the authors of the present paper would be able to distinguish between them for the analysis performed.

We agree with the authors that a randomized controlled trial comparing direct and indirect bypass for moyamoya disease would be the best method for determining the superiority of revascularization technique, but it is unlikely this will be done. However, the recent publication of the prospective, randomized controlled study, the Japan Adult Moyamoya Trial, comparing clinical outcomes of direct extracranial-intracranial bypass and no surgery for patients with hemorrhagic moyamoya disease, demonstrated a statistically significant benefit for direct revascularization in terms of both recurrent bleed rate and all morbidity. ${ }^{21}$ In the absence of a similar randomized trial comparing indirect bypass with no surgery, the procedure of choice for hemorrhagic moyamoya disease should be direct bypass.

A very recent study from Japan ${ }^{12}$ that very elegantly addressed a similar topic included 358 revascularizations in 236 patients (adults and pediatrics), together with a systematic review of 2032 cases of direct/combined revascularization and 4171 cases of indirect revascularization. The authors concluded that the postoperative stroke rate was comparable between the different surgical strategies but that the recurrent stroke risk was higher in the indirect bypass group. Despite the fact that direct bypass is more technically challenging than indirect bypass, experienced centers that frequently perform direct anastomoses become proficient with the direct technique. Therefore, at Stanford and many other centers, direct bypass is still the preferred revascularization strategy for most patients with moyamoya disease. However, in young children (who possess robust angioplasticity) and in other select cases in which no suitable donor or recipient arteries are available, indirect procedures remain our treatment of choice.

https://thejns.org/doi/abs/10.3171/2015.10.JNS152025

\section{References}

1. Abla AA, Gandhoke G, Clark JC, Oppenlander ME, Velat GJ, Zabramski JM, et al: Surgical outcomes for moyamoya angiopathy at Barrow Neurological Institute with comparison of adult indirect encephaloduroarteriosynangiosis bypass, adult direct superficial temporal artery-to-middle cerebral artery bypass, and pediatric bypass: 154 revascularization surgeries in 140 affected hemispheres. Neurosurgery 73:430-439, 2013

2. Arias EJ, Dunn GP, Washington CW, Derdeyn CP, Chicoine MR, Grubb RL Jr, et al: Surgical revascularization in North American adults with moyamoya phenomenon: long-term angiographic follow-up. J Stroke Cerebrovasc Dis 24:15971608,2015

3. Bang JS, Kwon OK, Kim JE, Kang HS, Park H, Cho SY, et al: Quantitative angiographic comparison with the OSIRIS program between the direct and indirect revascularization modalities in adult moyamoya disease. Neurosurgery 70:625-633, 2012

4. Burke GM, Burke AM, Sherma AK, Hurley MC, Batjer HH, Bendok BR: Moyamoya disease: a summary. Neurosurg Focus 26(4):E11, 2009

5. Czabanka M, Peña-Tapia P, Scharf J, Schubert GA, Münch E, Horn P, et al: Characterization of direct and indirect cerebral revascularization for the treatment of European patients with moyamoya disease. Cerebrovasc Dis 32:361-369, 2011

6. Duan L, Bao XY, Yang WZ, Shi WC, Li DS, Zhang ZS, et al: Moyamoya disease in China: its clinical features and outcomes. Stroke 43:56-60, 2012

7. Golby AJ, Marks MP, Thompson RC, Steinberg GK: Direct and combined revascularization in pediatric moyamoya disease. Neurosurgery 45:50-60, 1999

8. Guzman R, Lee M, Achrol A, Bell-Stephens T, Kelly M, Do $\mathrm{HM}$, et al: Clinical outcome after 450 revascularization procedures for moyamoya disease. Clinical article. J Neurosurg 111:927-935, 2009

9. Guzman R, Steinberg GK: Direct bypass techniques for the treatment of pediatric moyamoya disease. Neurosurg Clin $\mathbf{N}$ Am 21:565-573, 2010

10. Houkin K, Kuroda S, Ishikawa T, Abe H: Neovascularization (angiogenesis) after revascularization in moyamoya disease. Which technique is most useful for moyamoya disease? Acta Neurochir (Wien) 142:269-276, 2000

11. Kawaguchi S, Okuno S, Sakaki T: Effect of direct arterial bypass on the prevention of future stroke in patients with the hemorrhagic variety of moyamoya disease. J Neurosurg 93:397-401, 2000

12. Kazumata K, Ito M, Tokairin K, Ito Y, Houkin K, Nakayama $\mathrm{N}$, et al: The frequency of postoperative stroke in moyamoya disease following combined revascularization: a single-university series and systematic review. J Neurosurg 121:432-440, 2014

13. Khan N, Achrol AS, Guzman R, Burns TC, Dodd R, BellStephens T, et al: Sex differences in clinical presentation and treatment outcomes in moyamoya disease. Neurosurgery 71:587-593, 2012

14. Khan N, Schuknecht B, Boltshauser E, Capone A, Buck A, Imhof HG, et al: Moyamoya disease and moyamoya syndrome: experience in Europe; choice of revascularisation procedures. Acta Neurochir (Wien) 145:1061-1071, 2003

15. Kim CY, Wang KC, Kim SK, Chung YN, Kim HS, Cho BK: Encephaloduroarteriosynangiosis with bifrontal encephalogaleo(periosteal)synangiosis in the pediatric moyamoya disease: the surgical technique and its outcomes. Childs Nerv Syst 19:316-324, 2003

16. Kim DS, Kang SG, Yoo DS, Huh PW, Cho KS, Park CK: Surgical results in pediatric moyamoya disease: angiographic revascularization and the clinical results. Clin Neurol Neurosurg 109:125-131, 2007

17. Kim SK, Cho BK, Phi JH, Lee JY, Chae JH, Kim KJ, et al: Pediatric moyamoya disease: an analysis of 410 consecutive cases. Ann Neurol 68:92-101, 2010

18. Kim SK, Wang KC, Kim DG, Paek SH, Chung HT, Han $\mathrm{MH}$, et al: Clinical feature and outcome of pediatric cerebrovascular disease: a neurosurgical series. Childs Nerv Syst 16:421-428, 2000

19. Kim SK, Wang KC, Kim IO, Lee DS, Cho BK: Combined encephaloduroarteriosynangiosis and bifrontal encephalogaleo(periosteal)synangiosis in pediatric moyamoya disease. Neurosurgery 50:88-96, 2002

20. Macyszyn L, Attiah M, Ma TS, Ali Z, Faught R, Hossain $A$, et al: Direct versus indirect revascularization procedures for moyamoya disease: a comparative effectiveness study. J Neurosurg [epub ahead of print July 29, 2016. DOI: 10.3171/2015.8.JNS15504] 
21. Miyamoto S, Yoshimoto T, Hashimoto N, Okada Y, Tsuji I, Tominaga T, et al: Effects of extracranial-intracranial bypass for patients with hemorrhagic moyamoya disease: results of the Japan Adult Moyamoya Trial. Stroke 45:1415-1421, 2014

\section{Disclosures}

The authors report no conflict of interest.

\section{Response}

\section{Sherman C. Stein, MD, and Eric L. Zager, MD}

Department of Neurosurgery, Perelman School of Medicine, University of Pennsylvania, Philadelphia, Pennsylvania

We thank Dr. Teo and colleagues for their careful review of our manuscript and their thoughtful remarks. Their great success with direct revascularization in moyamoya disease gives them considerable authority on the subject. Unfortunately, many other centers have not achieved the same outcomes, as is evident from the literature.

As they point out, the direct approach has several advantages. Our model concentrates on perioperative complications and on late-onset ischemic stroke and hemorrhage as contributors to overall quality of life. Whatever effects of collateralization, cerebral blood flow levels, and reserve capacity have on quality of life cannot be quantified and, therefore, have not been included in the model.

Our model did not find significant increases in the rates of stroke or hemorrhage over time. There were no significant trends for any of the groups studied-that is, adults with direct or indirect revascularization or children with direct, indirect, or combined revascularization. This may represent relatively small numbers of observations in each subgroup, the relatively short follow-up periods published, or a true lack of increased risk.

We thank Dr. Teo and coworkers for identifying our misstatement that "indirect revascularization results in over one-half a QALY..." The true difference between direct and indirect revascularization is much smaller. Nevertheless, the difference remains highly significant. Over
4 years, adults who have undergone direct procedures average losses of one-half a QALY; the loss after indirect surgery is $10 \%$ less.

Dr. Teo and colleagues' emphasis on late-onset stroke risk, to the exclusion of other factors, may be misplaced. It must be recalled that direct procedures result in more perioperative complications, one-sixth of which are fatal. Sequelae of perioperative complications last the entire duration of follow-up, whereas those of late-onset stroke occur, on average, midway through the follow-up period. Hence, the former have a greater impact on QALYs. For example, a perioperative mortality rate of $1 \%$ and a follow-up of 4 years reduce overall QALYs by $4 \%$.

As alleged, the series reported by Duan et al. ${ }^{1}$ made no distinction between direct and indirect procedures. However, since $97 \%$ of the procedures reported were indirect, we felt confident including data from the paper as indirect vascularization.

The Japan Adult Moyamoya Trial ${ }^{2}$ showed that direct surgery is indeed superior to medical treatment. However, this trial cannot be invoked to compare direct and indirect revascularization. Nor can absence of a direct comparison say anything about the relative merits of the two approaches. Indeed, it serves as a justification for this analysis.

We do not dispute that, in the hands of a talented and experienced group such as that at Stanford, direct revascularization may be the better approach to treating moyamoya disease in adults. Nevertheless, current literature supports the indirect approach. As other neurosurgical centers acquire the same expertise, the subject should be revisited.

\section{References}

1. Duan L, Bao XY, Yang WZ, Shi WC, Li DS, Zhang ZS, et al: Moyamoya disease in China: its clinical features and outcomes. Stroke 43:56-60, 2012

2. Miyamoto S, Yoshimoto T, Hashimoto N, Okada Y, Tsuji I, Tominaga $\mathrm{T}$, et al: Effects of extracranial-intracranial bypass for patients with hemorrhagic moyamoya disease: results of the Japan Adult Moyamoya Trial. Stroke 45:1415-1421, 2014 